\title{
Viscoelastic Behavior of Multiwalled Carbon Nanotubes into Phenolic Resin
}

\author{
Edson Cocchieri Botelho ${ }^{\mathrm{a}, \mathrm{b} *}$, Michelle Leali Costa ${ }^{\mathrm{a}}$, Carlos Isidoro Braga ${ }^{\mathrm{a}}$, \\ Thomas Burkhart ${ }^{\mathrm{b}}$ Bernd Lauke
}

\author{
${ }^{a}$ Materials and Technology Department, São Paulo State University - UNESP, Guaratinguetá, SP, Brazil \\ ${ }^{\mathrm{b}}$ Institut für Verbundwerkstoffe GmbH, Kaiserslautern, Germany \\ 'Leibniz-Institut für Polymerforschung, Dresden, Germany
}

Received: January 9, 2012; Revised: November 10, 2012

\begin{abstract}
Nanostructured polymer composites have opened up new perspectives for multi-functional materials. In particular, carbon nanotubes (CNTs) have the potential applications in order to improve mechanical and electrical performance in composites with aerospace application. This study focuses on the viscoelastic evaluation of phenolic resin reinforced carbon nanotubes, processed by using two techniques: aqueous-surfactant solution and three roll calender (TRC) process. According to our results a relative small amount of CNTs in a phenolic resin matrix is capable of enhancing the viscoelastic properties significantly and to modify the thermal stability. Also has been observed that when is used TRC process, the incorporation and distribution of CNT into phenolic resin is more effective when compared with aqueous solution dispersion process
\end{abstract}

Keywords: carbon nanotubes, phenolic nanocomposites, rheology

\section{Introduction}

Over recent years composite materials, especially those involving carbon structures as reinforcement inside a polymer matrix, have become the focus of considerable research ${ }^{1-3} \cdot$ In particular, the exceptional properties reported for carbon nanotubes (CNT) have motivated the development of new nanotube-based composites materials ${ }^{4-6}$. In addition to their unique mechanical properties, which combined with their high aspect ratio and nanoscale dimensions makes them excellent candidates for composite reinforcement they also possess superior thermal and electrical properties ${ }^{7-9}$.

Many thermoplastic polymers and resins have been used to produce polymer/CNT materials which were proved to exhibit desirably improvement on properties. However, most polymers of researches on this subject are thermoplastics ${ }^{8-12}$ and few studies have been focused on thermosets (especially epoxy resins) and thereby less data of structure-property on phenolic resin has been accumulated.

Nowadays, phenolic resins are indeed irreplaceable materials for selective high-technology applications, offering high reliability under severe conditions. Because of its excellent ablative properties, structural integrity, thermal stability and solvent resistance, phenolic resins are still widely used, especially in thermal insulation materials, molding compounds, coatings and composite materials ${ }^{11-13}$.

Although significant advances have been made in recent years to overcome difficulties with the manufacture of polymer nanocomposites, processing remains a key challenge in fully utilizing the properties of the nanoscale reinforcement. A primary difficulty is achieving a good dispersion of the nanoscale filler in a composite, independent

*e-mail: ebotelho@feg.unesp.br of filler shape and aspect ratio. Without proper dispersion, filler aggregates tend to act as defect sites which limit the mechanical performance ${ }^{14-16}$.

Carbon nanotubes can be dispersed in water when is used appropriate surfactants, preferentially with those that have relatively high HLB (Hydrophyle-Lyphophyle Balance). The properties of surfactant such as its nature, its concentration, and type of interaction are known to play a very important role in the dispersion of carbon nanotubes ${ }^{4}$. The knowledge of the surface charge of carbon nanotubes is absolutely essential for understanding the interaction mechanism with ionic surfactants, and to predict colloidal stability of CNT solutions. Some works available in the literature ${ }^{4}$ showed that the CNT are negatively charged in water and some groups demonstrated insufficient debundling power of the anionic surfactant SDS due to charge repulsions, while others reported on uniform colloidal dispersion of SDS-coated $\mathrm{CNT}^{4}$. Self-assembly structures (hemicylinders) of adsorbed nonionic surfactants on graphite surfaces were shown to form strong alignment with the graphite symmetry axis ${ }^{4}$. In analogy to the model of epitaxial adsorption on graphite, the adsorption mechanism on nanotube walls (graphene) was suggested to produce specific self-organization of surfactant molecules (Figure 1). While previous works suggested the formation of SDS cylindrical micelles around $\mathrm{SWNT}^{4}$ (Figure 1a), helices or double helices ${ }^{4}$, as well as hemimicellar adsorption of a surfactant ${ }^{4}$ (Figure 1b). Also has been reported by the literature ${ }^{4}$ a structure less random adsorption with no preferential arrangement of the head and tail groups is responsible for the stabilization of the dispersions (Figure 1c). Among the ionic 

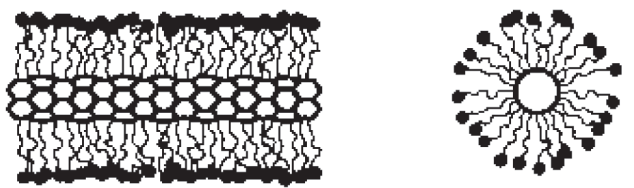

(a)

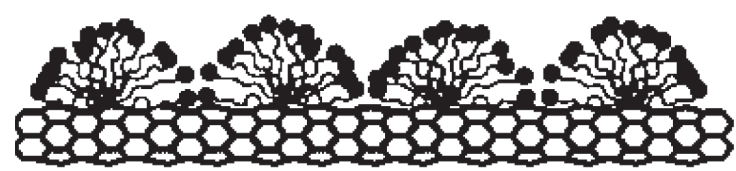

(b)

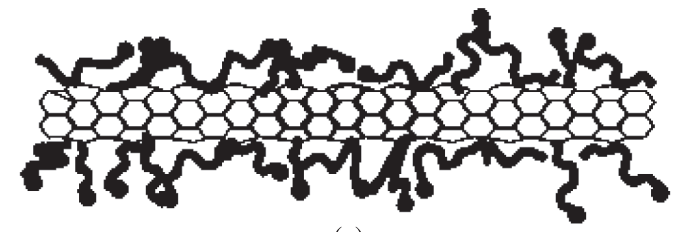

(c)

Figure 1. Models of the CNT/surfactant interaction: a) helices or double helices; b) hemimicellar adsorption of a surfactant; c) random adsorption ${ }^{4}$.

surfactants, SDS, poly (sodium 4-styrenesulfonate) sodium (PSSS) and dodecyl-benzene sodium sulfonate (NaDDBS $)^{4}$ were commonly used to decrease CNT aggregative tendency in water. Between them, the PSSS can be considered a better surfactant for a non-oxidized CNTs ${ }^{4}$.

Only relatively low concentrations $(<2-4$ wt. (\%)) of nanotubes can easily be incorporated in thermosetting composites, due to rapidly increasing viscosity and subsequent processing difficulties, at higher loadings. Even well-dispersed, shortened nanotubes can form a stiff gel, due to their high aspect ratio and resulting network-forming ability; the large interaction volume may also increase the background viscosity of the solvent/matrix ${ }^{17,18}$.

The rheological and mechanical properties of CNT-based composites also depend on the dispersion of nanotubes and interactions between nanotubes and polymer molecules. Therefore, understanding the rheological response of CNTs in fluids is of the most importance for design of polymer processing parameters. The rheological response of CNTs has been studied in a variety of polymer and polymer solutions but few works has been described from the literature involving CNT dispersion into phenolic resin by using three roll calender (TRC) processing ${ }^{19-22}$.

This study approaches two main objectives: firstly, the development of strategies to efficiently incorporate CNTs into phenolic resin, by using chemical and mechanical routs; secondly, to understand the viscoelastic behavior on phenolic resin when reinforced with different amounts of CNT.

\section{Methodology}

\subsection{Chemicals and materials}

The carbon nanotubes (Baytubes) used in this investigation has been supplied by Bayer Company (Germany). In this work was used, specifically, Baytubes
C $150 \mathrm{P}$ with an average diameter of $13-16 \mathrm{~nm}$, number of walls of 3-15 and bulk density of $140-160 \mathrm{~kg} \cdot \mathrm{m}^{-3}$. The manufacturer indicates that the CNTs contain less than 5\% impurities, including residual catalyst.

The dispersion of CNT into phenolic resin was achieved by using chemical and mechanical processing. In chemical way, it was used poly (sodium 4-styrenesulfonate) sodium (PSSS) surfactant from Sigma-Aldrich, in order to guarantee the dispersion of the CNTs in water.

Phenolic resin (SC 1008P) has been supplied by Hexion Company (Germany). This resin has as mainly characteristics: solid content around $66 \mathrm{wt}$. (\%) and gel time in $130{ }^{\circ} \mathrm{C}$ of around 18 minutes.

\subsection{Dispersion of CNTs into phenolic resin}

The dispersion of CNTs into phenolic resin was carried out by two ways: chemical and mechanical ways, as following:

- In the chemical way, first of all CNTs were dispersed into water with surfactant, by using a high intensity ultrasonic horn $(20 \mathrm{kHz}$, Branson PG), that was immerged into the liquid particle-resin-mixture. The vessel of the mixture was cooled in an ice water bath. During this method, we found that the ease of dispersal increased with sonification time: while unsonicated CNTs could not be dispersed in water, only 1 minute of sonification permitted a good dispersion. The ratio between the CNT and surfactant was 2 . This ratio was choosing according to the literature ${ }^{4-7,10}$. In order to mix the CNT dispersion into phenolic resin, both was processed using a mechanical mixer set up at a speed of 2,000 rpm.

- In the mechanical way, the masterbatch of CNTs was prepared by directly adding the nanofiber power to the liquid phenolic resin at room on a dissolver mixer. All masterbatchs was mechanically stirred under vacuum for about 45 minutes to remove most of the entrapped air introduced with the addition of the CNTs and to form a homogeneous suspension. In this mix process, has been used a speed of 2,000 rpm. After this first set, this masterbatch was dispersed with a three roll calender device (TRC), model Exakt $80 \mathrm{E}$. The dispersion took place in 3 steps, where the distance between the calender rolls was set up 150, 50 and $10 \mu \mathrm{m}$. The rotation speed of the cylinders was $150 \mathrm{rpm}$.

\subsection{Rheological and DMTA measurements}

Rheological measurements were performed at $25^{\circ} \mathrm{C}$ on a Rheometric Scientific Ares parallel plate rheometer with a torque transducer range of 0.004-100 g.cm, using $45 \mathrm{~mm}$ diameter parallel plates separated $1.5 \mathrm{~mm}$. All oscillatory measurements were performed within the linear viscoelastic regime which was experimentally determined using controlled frequency, variable strain measurements.

For the dynamical-mechanical analyses (DMTA) the masterbaths were cured in autoclave by using an appropriate curing cycle (Figure 2). The autoclave parameters used were: pressure of $7 \mathrm{~atm}$ and vacuum of $10^{-3} \mathrm{~atm}$. The surface of the cured specimens was also examined under SEM (scanning electron microscopy) technique, using a LEO 435 (Carl-Zeiss AG Oberkochen, Germany). The composite strands were fractured and the surface was observed. 
After cured, the specimens were cut at $4 \mathrm{~mm}$ width by $10 \mathrm{~mm}$ large and $50 \mathrm{~mm}$ longer and testes using a tension clamp, at a frequency of $1 \mathrm{~Hz}$, from 10 to $250{ }^{\circ} \mathrm{C}$, with a heating rate of $2{ }^{\circ} \mathrm{C} / \mathrm{min}$. From these tests viscoelastic parameters such as the $\tan \delta$, the loss E" and the storage E' moduli, and the glass transition temperature could be estimated.

\section{Results and Discussion}

\subsection{Viscosity of the Phenolic-CNT as a function of dispersion}

The commonly improvements in the mechanical and electrical properties of carbon nanotubes reinforced polymers can be generally related to the formation of a nanofiler microstructure within the polymer matrix. The formation of such microstructure can be investigated in the polymer in liquid state by using rheological experiments. Linear and non linear viscoelastic provide important information about the dispersion of the nanotubes wettability and the resulting structure-property relationship. This

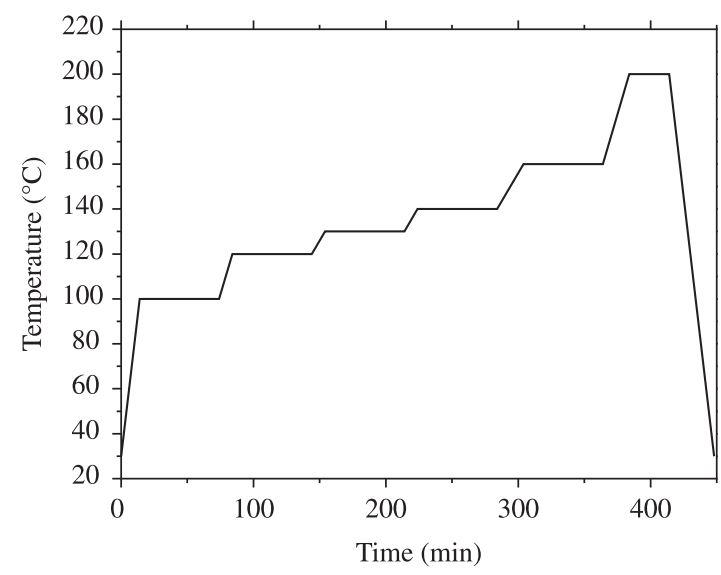

Figure 2. Cure cycle used in phenolic resin/CNT processing.

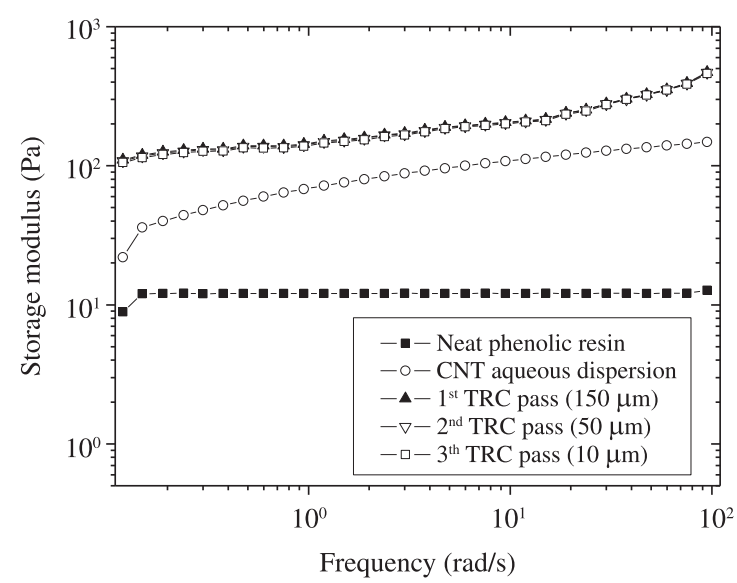

(a) information can also be used in order to improve the processability of the phenolic resin nanocomposites.

Figure 3 shows the storage (Figure $3 a$ ) and loss (Figure 3b) moduli, of the neat phenolic resin and of the 0.5 wt. (\%) CNT dispersion after to be used aqueous solution and passes into the TRC. As it can be observed and has also been reported in the literature ${ }^{19-22}$, the storage modulus is sensible to the reinforcement resulting from the added CNTs especially in the low frequency range. Storage modulus has increased with the incorporation of the CNTs into the polymer matrix; this enhancement was significantly higher as lower frequencies. It happens due to the CNT tend to form a percolated structure due to their high aspect ratio and surface area. The increase in modulus after the first pass in the three roll calender compared with the dissolver dispersed mixture can also be observed. This effect can be explained as a consequence of an increase in the interfacial area between the particles and the polymer resulting from the effective deagglomeration of the CNT agglomerates ${ }^{1-5}$. At low concentrations, the storage modulus enhancements is usually attributed to stiffness imparted by the solid particles that allow efficient stress transfer, which is mainly controlled by the interface interaction of CNT/matrix.

It can also be observed that the storage modulus showed a frequency independent solid-like behavior at low frequency, showing the appearance of a plateau, an effect that increased with the improved dispersion of the CNTs in the resin, this suggests the formation of some interconnected nanotubes network within the matrix. This is in accordance with Theoretical expectations and experimental observations for fiber-reinforced composites ${ }^{19-22}$.

The complex viscosity is also an important parameter to characterize the rheological properties of a material. The real part of the complex viscosity is an energy dissipation term similar to the imaginary part of the complex modulus. The dependency of the melt state complex viscosity of the neat epoxy resin and the 0.5 wt. (\%) CNT dispersion on frequency and passes through the TRC is shown in Figure 4.

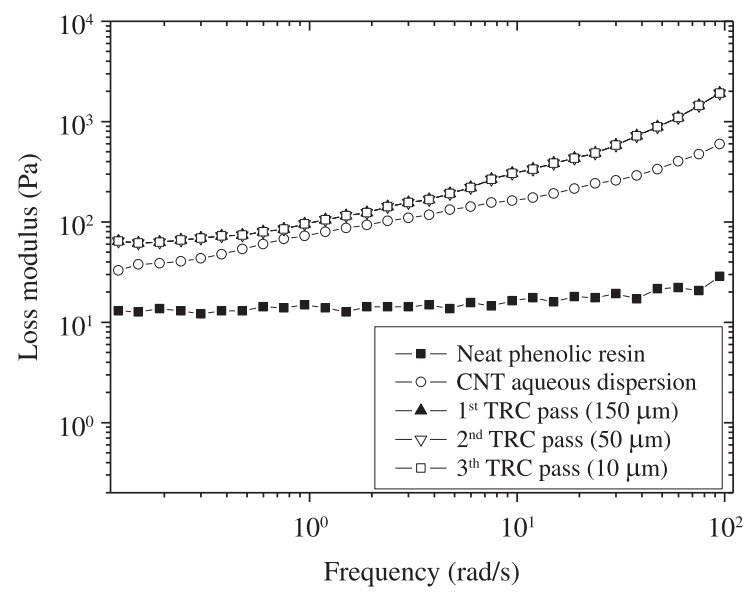

(b)

Figure 3. Storage and loss moduli against frequency for the phenolic-resin reinforced with CNT 0.1 wt. (\%) CNT: a) storage modulus and b) loss modulus evaluation. 
The neat phenolic resin showed a typical Newtonian behavior where the relatively low viscosity (around $5 \mathrm{~Pa}$ ) of the resin was independent of the frequency of the applied stress. On the other hand, the CNT reinforced composites exhibited a typical pseudo-plastic shear thinning behavior. As it can be seen in the graph, viscosity dramatically

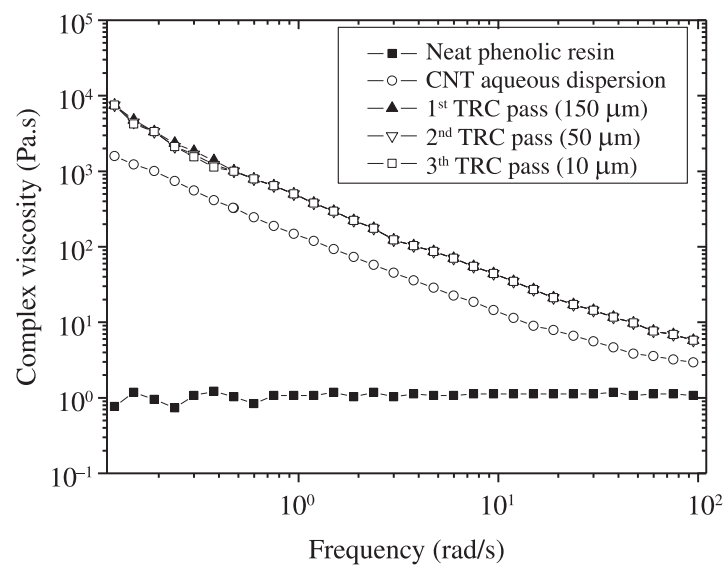

Figure 4. Complex viscosity against frequency for the phenolic-resin reinforced with CNT 0.5 wt. (\%) CNT.

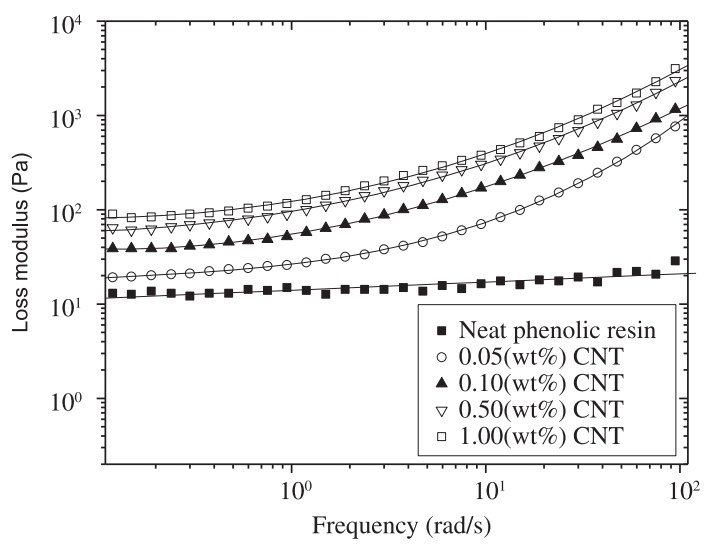

(a) increased with the presence of nanotubes and with dispersion through the calender and decreased with the increase in frequency. The steps passes through the TRC did not seem to have a significant effect on the viscosity of the mixture. In a filled polymer system the presence of CNT perturbs the normal flow of the polymer and hinders the mobility of the polymer chain segments in flow, therefore the viscosity of the polymer system increases.

It can also be observed that the composite became more shear dependent in the low frequency range. This shear thinning behavior can be attributed to an effective break up to the CNT agglomerates, leading to a greater degree of polymer-CNT interaction. These interactions require high shear stresses and longer relaxation times for the polymer to flow, thus the observed increase in viscosity.

\subsection{Viscoelastic behavior}

Figure 5 shows the viscoelastic behavior of CNT dispersion at concentrations ranging from 0.05 to 1.0 wt. (\%), dispersed by using TRC (after 3 steps). Typical response curves used for linear viscoelastic study when is considering strain limit are obtained before these tests only in order to evaluate the critical strain limit of the

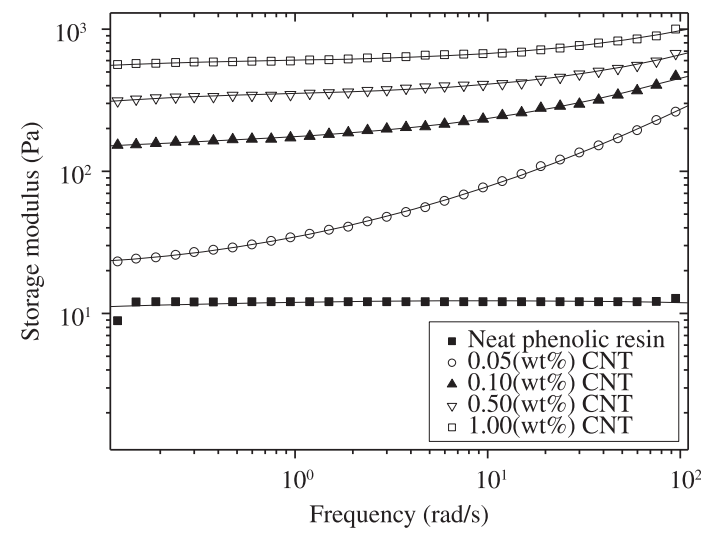

(b)

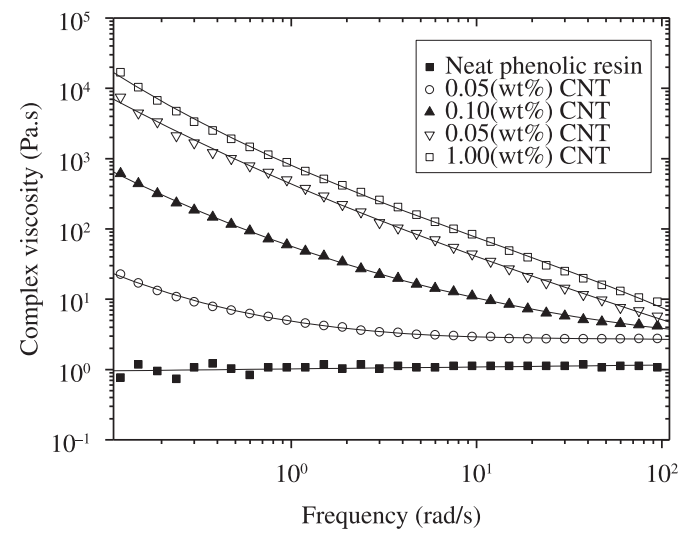

(c)

Figure 5. Phenolic resin reinforced CNT viscoelastic behavior: a) storage modulus evaluation; b) loss modulus evaluation and c) complex viscosity behavior. 
linear viscoelastic regime. According to these curves, for all wt. (\%) CNT content can be used $200 \mathrm{~Pa}$ as strain value.

A plot of the frequency-dependent storage modulus is shown in Figure 5a. In this case, the magnitude of G' increases with loading, accompanied by a flattening in the slope of response. This frequency-independent behavior at low $\omega$ is indicative of increasing solid like elastic response. In addition, the distinct jump in the low-frequency magnitude of $\mathrm{G}^{\prime}$ between 0.05 and $0.10 \mathrm{wt}$. (\%) is an indicative of percolation behavior. This jump is also reflected in the low-frequency loss modulus (Figure 5b). The complex viscosity is displayed by Figure 4c. In this case, the transition in behavior is evidenced by both an enhanced resistance to flow and increased degree of shear thinning.

\subsection{Rheological percolation}

A quantitative understanding of the critical concentration $\phi_{\mathrm{c}}$ required for nanotube percolation is necessary in order to optimize the balance between nanotube loading and mechanical properties. Significantly below $\phi_{c}$ there is insufficient fluid structure to provide considerable mechanical and electrical properties enhancement. The previously described jumps in $\mathrm{G}^{\prime}$ and $\eta^{*}$ between 0.05 and $0.10 \mathrm{wt}$. (\%) provide a first approximation for $\phi_{\mathrm{c}}$. Near the percolation threshold, the following power law can be used:

$\mathrm{G}^{\prime} \approx\left(\phi-\phi_{\mathrm{c}}\right)^{\mathrm{n}} / \phi_{\mathrm{c}}$

Where: $\phi$ is the CNT loading and $\mathrm{n}$ is the scaling exponent constant and both can be obtain from Figure 6 . The inset of Figure 5 shows data taken at $\omega=0.1$ graphically fit to the power law by maximizing the regression coefficient $\mathrm{R}^{2}$, resulting in $\phi_{c}$ and $n$ values as $0.08 \%$ and 3.33 , respectively. Similar results have been obtained by using $\omega=0.2$ and $0.5 \mathrm{rad} / \mathrm{s}$.

In the present case, the exponent may be influenced by the presence of CNT-rich phase and another works from the literature shows the same evidences ${ }^{22}$. The low shear aggregation tendency of some low-concentration CNT dispersions described in the subsequent section would also be consistent with the presence of a CNT-rich phase.

\subsection{DMTA analyses}

SEM was used to characterize the dispersion of the nanotubes into the phenolic resin. Representative micrographs are shown in Figure 7. The SEM images shows a fracture surface for phenolic resin with $0.5 \mathrm{wt}$. (\%) CNT, processed by aqueous solution (Figure 7a) and TRC by using the 3 steps (Figure 7b). In these cases it was observed a relatively homogeneous distribution of CNTs, but also there are some CNTs agglomerations mainly in the specimens processed in aqueous solution (Figure 7a).

The morphology also shows random orientation of CNTs into composites. Long CNTs on fracture surface are probably due to the weak interfacial strength between CNT and matrix.

Good dispersion of CNT in composites reduces the stress concentration and enhances the uniformity of stress distribution; as a result, the phenolic resin/CNT processed by TRC have a better performance in mechanical and viscoelastic properties than these of the dispersed in aqueous solution.
Figure 8 shows representative DMTA scans for phenolic resin /CNT composites obtained from CNT aqueous dispersion and TRC processes. As can be observed from Figures $8 \mathrm{a}$ and $8 \mathrm{~b}$, there is a significant difference in E' and E" moduli when compared the phenolic resin/CNT processed by using TRC or aqueous solution. According to

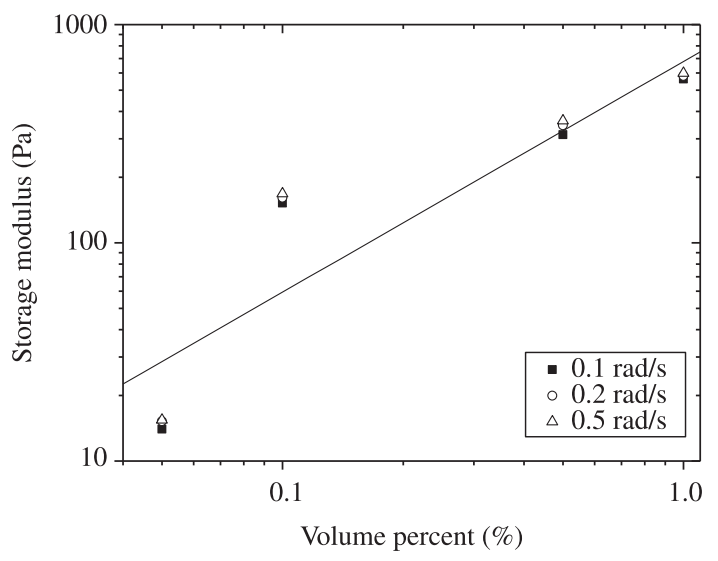

Figure 6. Storage modulus with varying CNT loading plotted at angular frequencies of $0.1,0.2$ and $0.5 \mathrm{rad} / \mathrm{s}$.

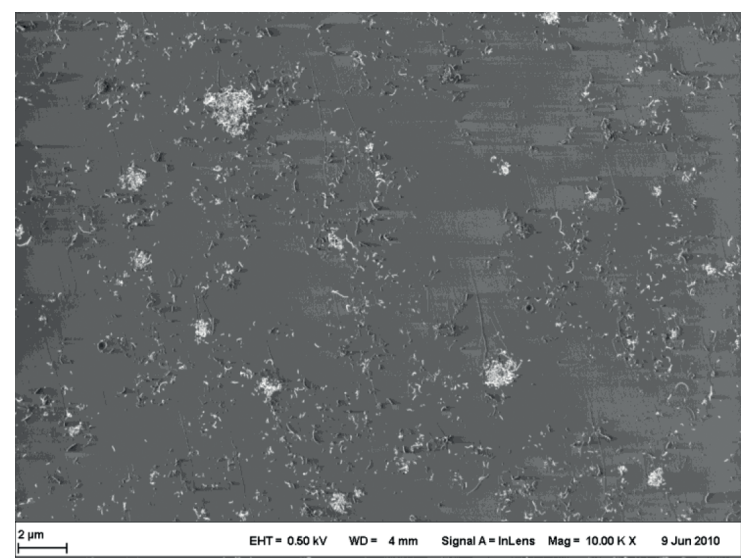

(a)

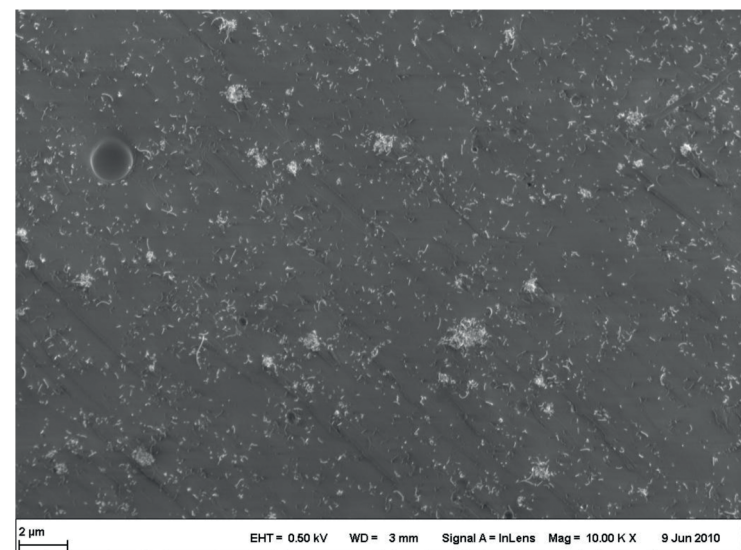

(b)

Figure 7. SEM micrographs from fractured surfaces of phenolic resin/CNT (0.5 wt. (\%) CNT content) composites: a) CNT aqueous solution and b) TRC. 
Figure $8 \mathrm{a}$, the initial rubbery plateau modulus (around $30^{\circ} \mathrm{C}$ ) is twice higher for the nanostructured composite obtained with TRC. A similar results could be observed with the E" in glass transition temperature region (Figure $8 \mathrm{~b}$, in around $70{ }^{\circ} \mathrm{C}$ ). Several factors may contribute to the increased rubbery plateau modulus including: dispersion reinforcing effect by the nanotubes, increased crosslink density and restricted mobility from enhanced polymer nanotube interactions. However, many studies on thermoset/CNT nanocomposites have reported significant glassy modulus enhancements, with no rubbery modulus enhancements ${ }^{20-22}$.

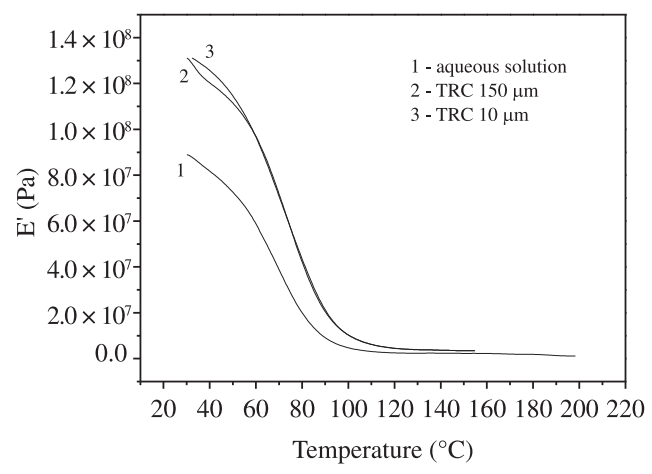

(a)

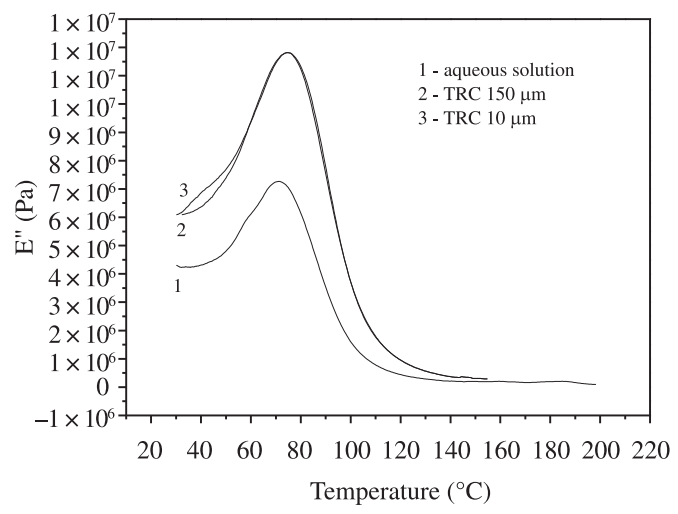

(b)

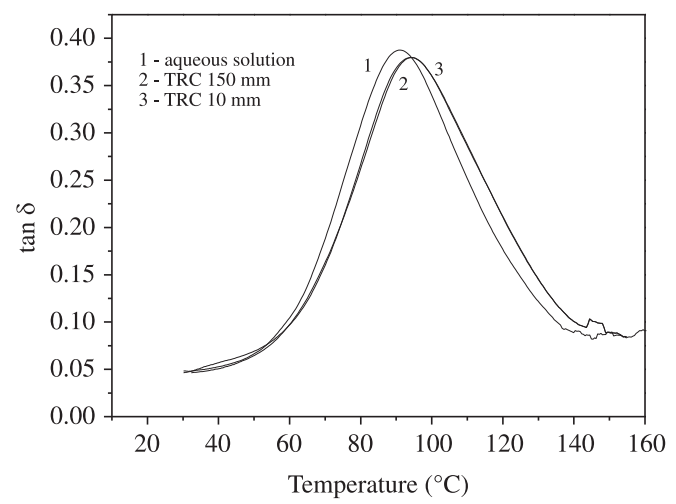

(c)

Figure 8. DMA results for nanostructured composites processed with phenolic resin and CNT (0.1 wt. (\%) CNT): (a) E', (b) E" and (c) tan delta.
Figure $9 \mathrm{a}$ and $9 \mathrm{~b}$ show the glass transition results obtained from tan delta, E' and E" curves, respectively. According to this study, the Tg values was not significantly affected by the crosslink between the reinforcement and the matrix, since in all cases the differences between the $\mathrm{Tg}$ observed was lower than $3{ }^{\circ} \mathrm{C}$. This figure also shows that there is no significant difference from the glass transition temperature when compared phenolic resin/CNT processed with more than one step in TRC and in specimens prepared by aqueous solution. Also in this case, the differences in $\mathrm{Tg}$ was lower than $3{ }^{\circ} \mathrm{C}$.

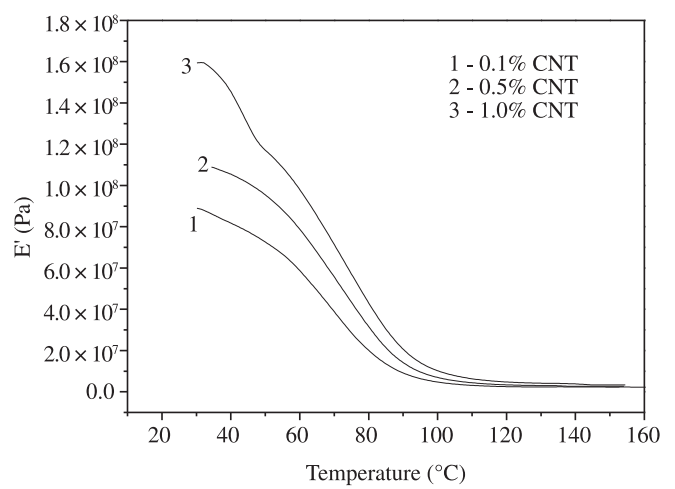

(a)

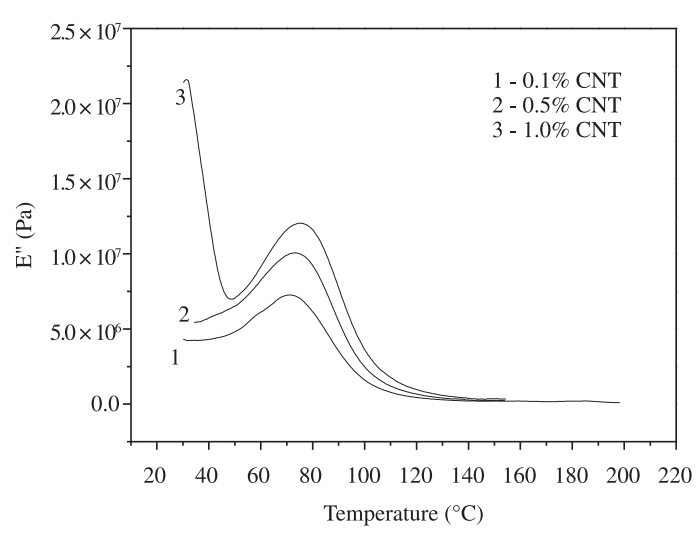

(b)

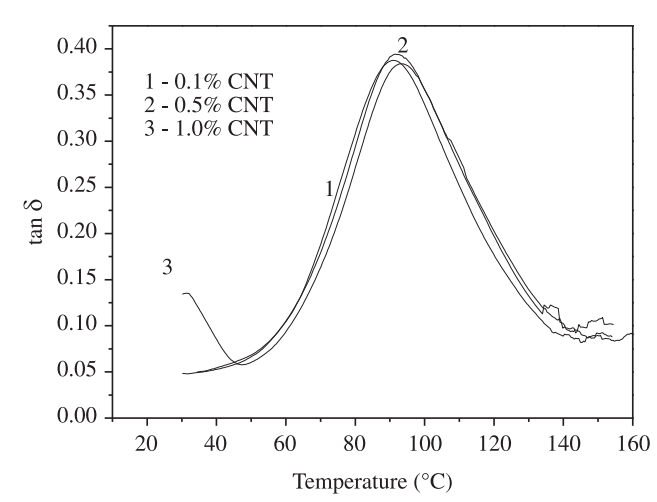

(c)

Figure 9. DMA evaluation for phenolic/CNT nanostructured composites with different wt. (\%) CNT content: (a) E', (b) E" and (c) tan delta. 


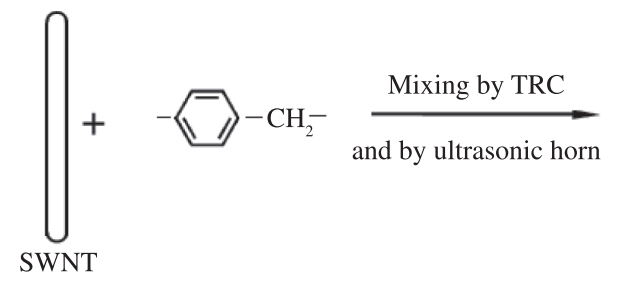

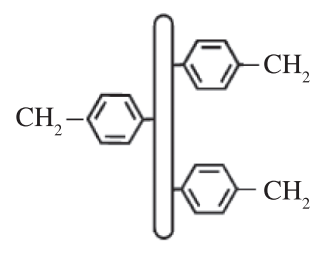

PR-SWNT

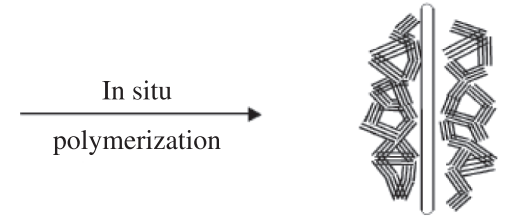

CPR-SWNT

Figure 10. Suggested mechanisms for the CNT/Phenolic resin interaction. SWNT (single walled nanotube); PR (phenolic resin); CPR (cured phenolic resin).

The effect of nanotube amount on the damping behavior is shown by the plot of tan delta versus temperature in Figures $8 \mathrm{c}$ and $9 \mathrm{c}$, where the molecular mobility and nanotube concentration are enhanced. According to the literature 4 there may be a CNT concentration gradient through the phenolic resin in the $\mathrm{Tg}$ region and a corresponding distribution of temperature dependent of the molecular relaxation time which may affect the DMA data. The loss factor (tan delta) are very sensitive to the structural transformation and also to the interface modification of the material produced on increasing the temperature and provide a very efficient means of analysis in the timescale of the relaxation. Also according to the literature ${ }^{2-10}$ free volume theories argue that the Tg of a polymer system decreases by an amount proportional to the amount of nanoparticles incorporation, and also after resin curing process (Figure 10). But, since CPR (cured phenolic resin) is a cross-linking polymer, it would be difficult to wrap it around an SWNT simply through noncovalent interactions. However, through covalent interactions it would be possible for PR to bond directly with the SWNTs and to wrap them. Therefore, no significant differences have been observed in tan delta values (Figures 8c and 9c). A similar behavior in local chain mobility due to the incorporation of nanoparticles has been found in other nanocomposites (e.g., epoxy/CNT). A possible explanation for this behavior is that there is no enouth confinement of the polymer in nanoscale spaces between the nanoparticles and low polymer-particle interaction.

\section{Conclusions}

The above results have demonstrated that a relative small amount of CNTs in a phenolic resin matrix is capable of enhancing the viscoelastic properties significantly and to modify the thermal stability. Also has been observed that when is used TRC process, the incorporation and distribution of CNT into phenolic resin is more effective when compared with aqueous solution dispersion process.

The resulting of rheological mastercurves showed two separate types of rheological response: at concentrations below the percolation threshold, the response suggested a developing nanotube network structure, whereas at higher concentrations a saturated and elastic network structure was observed. These observations were correlated with the onset of rheological percolation which was found to be comparable based on several methods, including a recently proposed technique of tracking the evolution of yield stress.

In addition to enhancing dispersability of the CNTs into phenolic resin, formation of covalent bond has a dramatic effect on the behavior of this thermoset resin, in both the uncured and cured state. For example, in the uncured state, the presence of the covalent bond affects the rheological behavior, resulting in a transition from Newtonian behavior for the neat resin to non-Newtonian behavior for the nanocomposites. In the solid state, the nature of the covalent interactions not affects the $\mathrm{Tg}$ relaxation but affect the glassy modulus as well as the rubbery plateau modulus.

\section{Acknowledgements}

The authors acknowledge financial support received from FAPESP (under grant 2009/06335-9) and CNPq under grants 306053/2006-3.

\section{References}

1. Abdalla M, Dean D, Adibempe D, Nyairo E, Robinson P and Thompson G. The effect of interfacial chemistry on molecular mobility and morphology of multiwalled carbon nanotubes epoxy nanocomposite. Polymer. 2007; 48(19):5662-70. http:// dx.doi.org/10.1016/j.polymer.2007.06.073

2. Grady BP. Recent Developments Concerning the Dispersion of Carbon Nanotubes in Polymers. Macromolecular Rapid Comunications. 2010; 31(3):247-57. PMid:21590898. http:// dx.doi.org/10.1002/marc.200900514
3. Diez-Pascual AM, Naffakh M, Gómez MA, Marco C, Ellis G, Martínez MT, et al. Development and characterization of PEEK/ carbon nanotube composites. Carbon. 2009; 47(13):3079-90. http://dx.doi.org/10.1016/j.carbon.2009.07.020

4. Vaisman L, Wagner HD and Marom G. The role of surfactants in dispersion of carbon nanotubes. Advances in Colloid and Interface Science. 2006; 128-130:37-46. PMid:17222381. http://dx.doi.org/10.1016/j.cis.2006.11.007

5. 5.Yu J, Grossiord N, Koning CE and Loos J. Controlling the dispersion of multi-wall carbon nanotubes in aqueous surfactant solution. Carbon. 2007; 45(3):618-623. http://dx.doi. org/10.1016/j.carbon.2006.10.010 
6. Moore VC, Strano MS, Haroz EH, Hauge RH, Smalley $\mathrm{RE}$, Schmidt J et al. Individually Suspended Single-Walled Carbon Nanotubes in Various Surfactants. Nano Letters. 2003; 3:1379-82. http://dx.doi.org/10.1021/n1034524j

7. Bal S and Samal SS. Carbon nanotube reinforced polymer composites - A state of the art. Bulletin of Material Science. 2007; 30(4):379-386. http://dx.doi.org/10.1007/ s12034-007-0061-2

8. Yang J, Xu T, Zhang Q, Lu A and Fu Q. Electrical properties of poly(phenylene sulfide)/multiwalled carbon nanotube composites prepared by simple mixing and compression. Journal of Applied Polymer Science. 2008; 109(2):720-26. http://dx.doi.org/10.1002/app.28098

9. Kotsilkova R, Ivanov E, Krusteva E, Silvestre C, Cimmino $\mathrm{S}$ and Duraccio D. Isotactic polypropylene composites reinforced with multiwall carbon nanotubes, part 2: Thermal and mechanical properties related to the structure. Journal of Applied Polymer Science. 2009; 115(6):3576-85. http://dx.doi. org/10.1002/app.30413

10. Angelikopoulos P, Gromov A, Leen A, Nerushev O, Bock H and Campbell EEB. Dispersing Individual Single-Wall Carbon Nanotubes in Aqueous Surfactant Solutions below the cmc. Journal of Physical Chemistry C. 2010; 114(1):2-9. http:// dx.doi.org/10.1021/jp905925r

11. Mathur RB, Singh BP, Dhami TL, Kalra Y, Lal N, Rao $\mathrm{R}$ et al. Influence of carbon nanotube dispersion on the mechanical properties of phenolic resin composites. Polymer Composites. 2010; 31(2):321-327. http://dx.doi.org/10.1002/ pc. 20807

12. Natali M, Kenny $J$ and Torre L. Phenolic matrix nanocomposites based on commercial grade resols: Synthesis and characterization. Composites Science and Technology. 2010; 70(4):571-577. http://dx.doi.org/10.1016/j. compscitech.2009.12.005

13. Chen GX, Hong MH, Ong TS, Lam HM, Chen WZ and Elim HI. Carbon nanoparticles based nonlinear optical liquid. Carbon. 2004; 42(12-13):2735-2777. http://dx.doi. org/10.1016/j.carbon.2004.05.035

14. Tai N-H, Yeh M-K and Peng T-H. Experimental study and theoretical analysis on the mechanical properties of SWNTs/ phenolic composites. Composites Part B. 2008; 39(6):926-932. http://dx.doi.org/10.1016/j.compositesb.2008.01.003

15. Tao K, Yang S, Grunlan JC, Kim Y-S, Dang B, Deng Y et al. Effects of carbon nanotube fillers on the curing processes of epoxy resin-based composites. Journal of Applied Polymer Science. 2006; 102:5248-5254. http://dx.doi.org/10.1002/ app. 24773

16. Ye C, Gang Q-M, Lu F-P and Liang J. Preparation of carbon nanotubes/phenolic-resin-derived activated carbon spheres for the removal of middle molecular weight toxins. Separation and Purification Technology. 2008; 61(1):9-14. http://dx.doi. org/10.1016/j.seppur.2007.09.021

17. Hwang HJ, Jung SL, Cho KH, Kim YJ and Jang H. Tribological performance of brake friction materials containing carbon nanotubes. Wear. 2010; 268(3-4):519-525. http://dx.doi. org/10.1016/j.wear.2009.09.003

18. Prashantha K, Soulestin J, Lacrampe MF, Claes M, Dupin G and Krawczak P. Multi-walled carbon nanotube filled polypropylene nanocomposites based on masterbatch route: Improvement of dispersion and mechanical properties through PP-g-MA addition. Express Polymer Letters. 2008; 2(10):735-745.

19. Ma AWK, Yearsley KM, Chinesta F and Mackley MR. A review of the microstructure and rheology of carbon nanotube suspensions. Journal of Nanoengineering and Nanosystems. 2009; 222:71-94. http://dx.doi. org/10.1243/17403499JNN153

20. Velasco-Santos C, Martínez-Hernández AL, Fisher F, Ruoff R and Castaño VM. Dynamical-mechanical and thermal analysis of carbon nanotube-methyl-ethyl methacrylate nanocomposites. Journal of Physics D: Applied Physics. 2003; 36(12):1423-28. http://dx.doi.org/10.1088/0022-3727/36/12/311

21. Huang YY, Ahir SV and Terentjev EM. Dispersion rheology of carbon nanotubes in a polymer matrix. Physical Review B. 2006; 73:125422-1-9. http://dx.doi.org/10.1103/ PhysRevB.73.125422

22. Kayatin MJ and Davis VA. Viscoelasticity and Shear Stability of Single-Walled Carbon Nanotube/Unsaturated Polyester Resin Dispersions. Macromolecules. 2009; 42(17):6624-6632. http:// dx.doi.org/10.1021/ma901010d 